\title{
Langerhans cell protein 1 (LCP1) binds to PNUTS in the nucleus: implications for this complex in transcriptional regulation
}

\author{
Shin-Jeong Lee ${ }^{1}$, Jun-Ki Lee ${ }^{1}$, \\ Yong-Sun Maeng ${ }^{1}$, Young-Myeong Kim ${ }^{2}$ \\ and Young-Guen Kwon ${ }^{1,3}$ \\ 'Department of Biochemistry \\ College of Life Science and Biotechnology \\ Yonsei University \\ Seoul 120-752, Korea \\ ${ }^{2}$ Department of Molecular and Cellular Biochemistry \\ School of Medicine, Kangwon National University \\ Chunchon 200-701, Korea \\ ${ }^{3}$ Corresponding author: Tel, 82-2-2123-5697; \\ Fax, 82-2-362-9897; E-mail, ygkwon@yonsei.ac.kr \\ DOI 10.3858/emm.2009.41.3.022
}

Accepted 18 November 2008

Abbreviations: GBD, GAL4 DNA binding region; LCP1, Langerhans cell protein 1; PNUTS, protein phosphatase 1 nuclear targeting subunit

\begin{abstract}
Protein phosphatase-1 (PP1) nuclear targeting subunit (PNUTS), also called PP1R10, p99, or CAT 53 was originally isolated as a mammalian nuclear PP1-binding protein. In this study, we performed yeast two-hybrid screens to identify PNUTS-interacting proteins. Here, we report that LCP1 (epidermal Langerhans cell protein 1), a novel member of the HMG-box protein family, binds tightly to PNUTS. Co-immunoprecipitation of deletion constructs revealed that the $\mathrm{C}$-terminus of LCP1 is sufficient for the interaction with an $\mathrm{N}$-terminal region of PNUTS that is distinct from its PP1-binding domain. Furthermore, immunofluorescence studies showed that a subpopulation of LCP1 co-localizes with PNUTS in nuclear speckles. Importantly, we found that the N-terminus of LCP1 has a strong trans-activation activity in a GAL4-based heterologous transcription assay. The transcriptional activity of LCP1 is markedly suppressed by its interaction with PNUTS, in a PP1-independent manner. These findings suggest that the coordinated spatial and temporal regulation of LCP1 and PNUTS may be a novel mechanism to control the expression of genes that are critical for certain physio-
\end{abstract}

logical and pathological processes.

Keywords: PPP1R10 protein, human; protein interaction mapping; transcription factors; two-hybrid system techniques

\section{Introduction}

Protein phosphatase-1 (PP1) nuclear targeting subunit (PNUTS), also known as PP1R10, p99, or CAT 53 , was originally isolated as a mammalian nuclear PP1-binding protein (Allen et al., 1998; Kim et al., 2003; Ruma et al., 2005). The human PNUTS homologue has been identified in the HLA class 1 region on the short arm of chromosome 6 , which has been linked to hereditary hemochromatosis (Ruma et al., 2005). The PNUTS protein has been detected in various tissues, at high levels in testis, brain, and intestine and low levels in heart and skeletal muscle (Allen et al., 1998). Biochemical analysis has shown that PNUTS binds to PP1 through a consensus PP1-binding 'RVXF motif' (398TVTW401) and to homopolymeric RNA, with high selectivity for poly(A) and $\operatorname{poly}(G)$, through a region of its $\mathrm{C}$-terminus that contains RGG motifs (Allen et al., 1998; Kim et al., 2003). Primary sequence analysis indicates that PNUTS possesses a transcription elongation factor SII domain in its $\mathrm{N}$-terminus and a single putative $\mathrm{Zn}^{2+}$ finger with the signature $\mathrm{C}-\mathrm{X} 8-\mathrm{C} \times 5-\mathrm{C}-\mathrm{X} 3-\mathrm{H}$ at its extreme C- terminus (Kim et al., 2003).

Several lines of evidence suggest that PNUTS functions in cell cycle progression and apoptosis: PNUTS inhibits PP1c activity towards the Retinoblastoma protein (pRb) (Udho et al., 2002; Krucher et al., 2006; De et al., 2008; Graña, 2008). Knockdown of PNUTS in MCF7, SKA, and HCT116 cancer cells leads to caspase-mediated apoptosis (De et al., 2008). We have demonstrated that PNUTS is a hypoxia-inducible gene that regulates the phosphorylation and apoptotic activities of p53 (Lee et al., 2007). Overexpression of PNUTS increases cell death in response to hypoxia through the increased expression of Bax, an apoptosisrelated gene induced by p53 as well as the ubiquitin-dependent proteosomal degradation of MDM2 (Lee et al., 2007). PNUTS is targeted to the 
nucleus in telophase, following the assembly of the nuclear membrane and concomitant with chromatin decondensation (Landsverk et al., 2005). In addition, PNUTS has been shown to augment in vitro chromosome decondensation in a PP1-dependent manner (Landsverk et al., 2005). PNUTS has also been suggested to have potential roles in the nervous system, with its expression predominantly localized to specific regions of the human and mouse brain (Ruma et al., 2005). In-situ hybridization and immunohistochemistry analysis has revealed high levels of PNUTS expression in the hippocampus, frontal, and entorhinal cortex of normal brains and in the neurofibrillary tangles of Alzheimer's diseased brains (Ruma et al., 2005). Recently, it has also been shown that PNUTS co-localizes with PP $1 \gamma 1$ and $\mathrm{GABA}_{c}$ receptors in the axon termini of rod bipolar cells where it forms a trimeric protein complex with these proteins, suggesting that PNUTS regulates PP1 at retinal synapses (Rose et al., 2008). However, despite these insights, to date the precise physiological role of PNUTS remains elusive.

There are many examples of PP1-targeting proteins that mediate the formation of protein complexes (Cohen, 2002). Often, these complexes function as signaling modules that are localized in close proximity to the substrates of associated protein kinases and phosphatases (Depaoli-Roach et al., 1994; Cohen, 2002). Therefore, the identification and characterization of PNUTS binding partners may illuminate the physiological function of PNUTS. In the present study, we have employed the yeast two-hybrid method to identify PNUTSinteracting proteins. We report that LCP1 (epidermal Langerhans cell protein 1), which represents a novel member of the HMG-box protein family, is tightly associated with PNUTS in the mammalian nucleus. Furthermore, this study defines the interaction domains of the two proteins and characterizes their potential roles in transcriptional regulation.

A

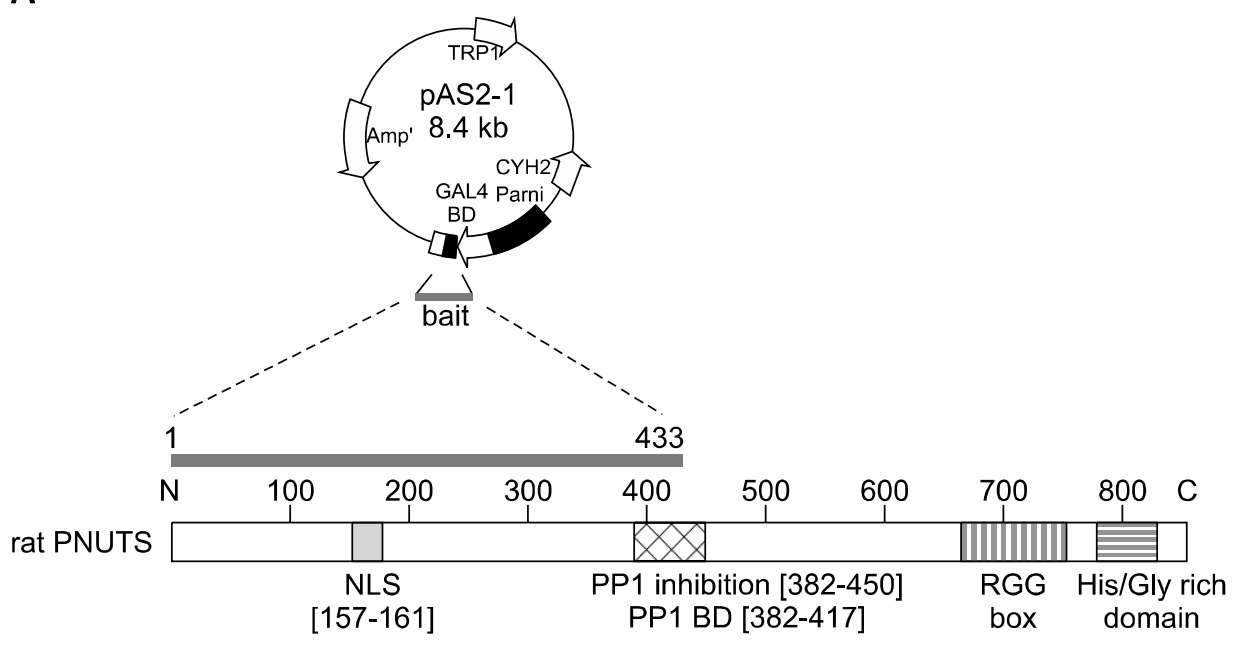

B

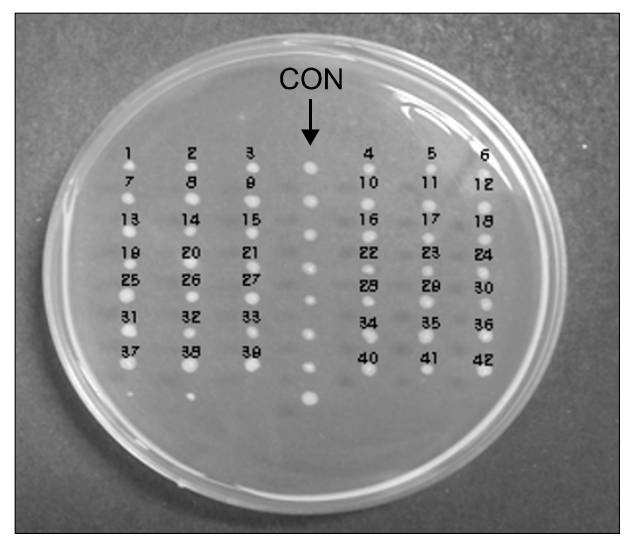

In SD (-Trp, -Leu) medium
C

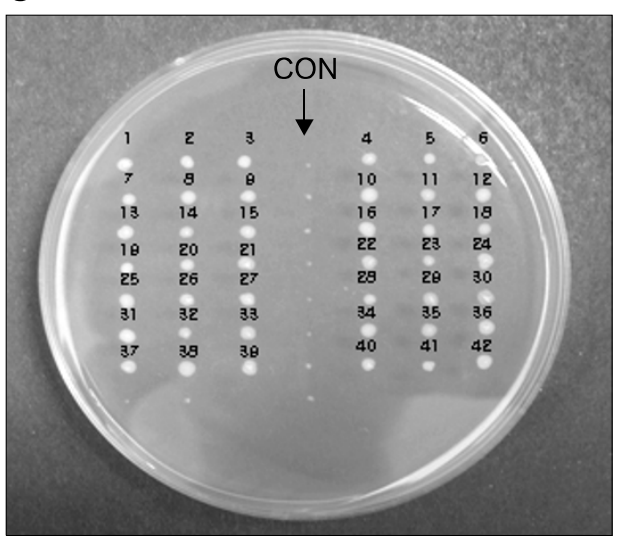

In SD (-Trp, -Leu, -His) medium
Figure 1. 42 positive clones were isolated from screening a human brain cDNA library with the GAL4BDPNUTS (1-433) bait plasmid. (A) Design of the bait plasmid, GAL4BDPNUTS (1-433) fusion construct, pAS2-1/PNUTS (1-433). pAS2-1 map; pAS2-1 containing the selectable marker, TRP1, is the cloning vector used to generate bait fusion proteins; a domain (residues 1-433) of PNUTS was inserted into pAS2-1. (B) Positive colonies and controls grew well in SD media lacking tryptophan and leucine. (C) Only positive colonies were able to grow in SD lacking tryptophan, leucine, and histidine. 


\section{Results}

\section{Identification of LCP1 as a PNUTS interacting protein by yeast two-hybrid}

To identify proteins that bind to PNUTS, we used the yeast two-hybrid method to screen a human brain cDNA-GAL4 expression plasmid library. The two-hybrid "bait" protein consisted of a fragment of PNUTS (1-433) fused to the GAL4 DNA-binding domain (Figure 1A). We screened approximately $3.0 \times 10^{6}$ yeast transformants and identified 42 primary candidates (Figure $1 \mathrm{~B}$ and $\mathrm{C}$ ). The corresponding prey plasmids were isolated and sequenced. The candidate PNUTS binding partners were then identified by NCBI BLAST searches of the nucleotide and protein databases. Two of the clones encoded full-length PP1C- $\alpha$ (protein phosphatase 1, catalytic subunit-alpha), a known PNUTS-binding protein. Interestingly, 36 of the 40 positive clones (with the exception of 4 clones which did not produce clean sequencing reads) encoded the same protein, KIAA0737, which shares $95 \%$ homology with murine epidermal Langerhans cell protein-1 (LCP1) (O'Flaherty and
Kaye, 2003). Of these, three clones (\#34, \#37, and \#39) were full length and the rest encoded various fragments of the KIAA0737 protein (Table 1). We selected the clone encoding KIAA0737 for further investigation. The full-length KIAA0737 clone

Table 1. The BLAST search results of yeast two-hybrid positive clones. Positive clones were sequenced and identified as encoding one of four genes. Two clones encoded full-length PP1C $\alpha, 36$ of 40 positive clones encoded KIAA0737, which has $95 \%$ homology with LCP1.

\begin{tabular}{ccc}
\hline $\begin{array}{c}\text { PNUTS } \\
\text { binding } \\
\text { protein }\end{array}$ & $\begin{array}{c}\text { The number } \\
\text { of positive clone }\end{array}$ & $\begin{array}{r}\text { The number } \\
\text { of LCP1 amino } \\
\text { acid }\end{array}$ \\
\hline KIAA0737 & $\# 37, \# 34, \# 39$ & $1-621$ a.a. \\
& $\# 1, \# 2, \# 3, \# 5, \# 13, \# 16$, & $107-621$ a.a. \\
& $\# 17, \# 19, \# 24, \# 26, \# 27$ & \\
& $\# 30, \# 36, \# 42$ & $127-621$ a.a. \\
& $\# 4, \# 7, \# 8, \# 9, \# 11, \# 14$, & $297-621$ a.a. \\
& $\# 15, \# 18, \# 20, \# 22, \# 23, \# 25$ & \\
$\# 6, \# 33, \# 38$ & $389-621$ a.a. \\
$\# 10, \# 29, \# 35$ & $415-621$ a.a. \\
& $\# 28$ & $538-621$ a.a. \\
\hline
\end{tabular}

\footnotetext{
1 atggagtttcccggaggaaatgacaatt acctgacgatcacagggecttcgc acccet tcctgt caggggccgagacat tcc at acacca $\begin{array}{lllllllllllllllllllllllllllllll}1 & \text { M } & \text { E } & \text { F } & \text { P } & \text { G } & \text { G } & \text { H } & \text { D } & \text { H } & \text { Y } & \text { L } & \text { T } & \text { I } & \text { T } & \text { G } & \text { P } & \text { S } & \text { H } & \text { P } & \text { F } & \text { L } & \text { S } & \text { G } & \text { A } & \text { E } & \text { T } & \text { F } & \text { H } & \text { T } & \text { P }\end{array}$ 91 agct tgggtgat gaggaatttgaaatcc cacctatctcct tggattct gatccetcat tggctgtctcagatgtggtt ggec actttgat

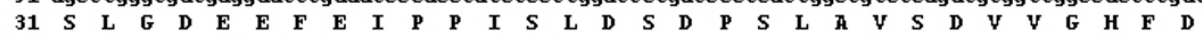

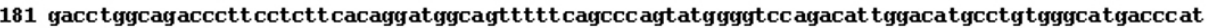

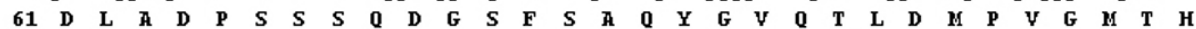
271 ggct tgat ggageagggceggggggctcct tgagt ggggget t tgaccat gyact t gyacc actctataggaactcagt at agt gccaaccca $\begin{array}{lllllllllllllllllllllllllllllll}91 & G & \text { L } & \text { M } & \text { E } & \text { O } & \text { G } & \text { G } & \text { G } & \text { L } & \text { L } & \text { S } & \text { G } & \text { G } & \text { L } & \text { T } & \text { M } & \text { D } & \text { L } & \text { D } & \text { H } & \text { S } & \text { I } & \text { G } & \text { T } & \text { O } & \text { Y } & \text { S } & \text { A } & \text { H } & \text { P }\end{array}$ 361 cctgttacaattgatgtaccaatgacagacatgacatctggcttgatgggge atagcc agtt gaccaccattgatcagtcagaact gagt $\begin{array}{llllllllllllllllllllllllllllllll}121 & \text { P } & \text { V } & \text { T } & \text { I } & \text { D } & \text { V } & \text { P } & \text { M } & \text { T } & \text { D } & \text { M } & \text { T } & S & \text { G } & \text { L } & \text { M } & \text { G } & \text { H } & S & \mathbf{0} & \text { L } & \text { T } & \text { T } & \text { I } & \text { D } & \text { O } & \text { S } & \text { E } & \text { L } & S\end{array}$ 451 tcccaget gggttt gagcctaggggggt ggcaccatcctgccacctgcccagt cacctgaagatcgtctttcaaccacccett cacctact $\begin{array}{lllllllllllllllllllllllllllllllll}151 & S & \mathbf{O} & \text { L } & \text { G } & \text { L } & \text { S } & \text { L } & \text { G } & \text { G } & \text { G } & \text { T } & \text { I } & \text { L } & \text { P } & \text { P } & \text { A } & \text { Q } & \text { S } & \text { P } & \text { E } & \text { D } & \text { R } & \text { L } & \text { S } & \text { T } & \text { T } & \text { P } & \text { S } & \text { P } & \text { T }\end{array}$

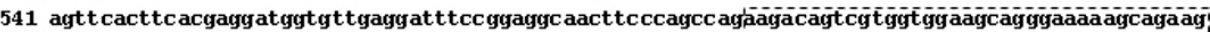

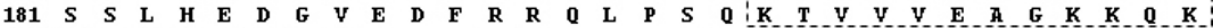

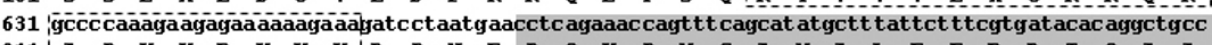

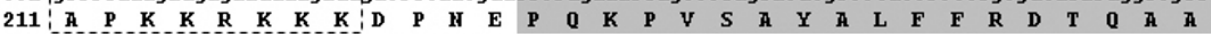
721 atcaagggacagaatcctaatgccactt ttggtgaggtttcaaaaattgtggcctccatgtgggatagtcttggagaggagc aaaaac ag

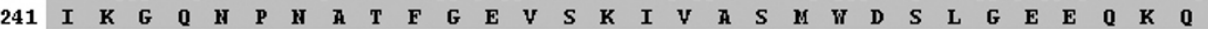
811 gtat ataagaggaaaactgaggctgccaagaaagagtatctgaaggcactggctgctt acaaagacaacc aggagt gtcaggccactgtg

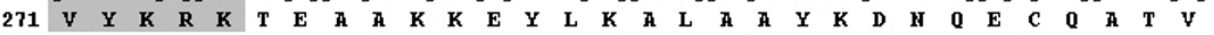
901 gaaacagtggaattggatccagcaccaccatcacaaactccttctccacctcctatggctactgttgacccagcatctccagcaccagct $\begin{array}{lllllllllllllllllllllllllllllll}301 & \mathbf{E} & \mathbf{T} & \mathbf{V} & \mathbf{E} & \mathbf{L} & \mathbf{D} & \mathbf{P} & \mathbf{A} & \mathbf{P} & \mathbf{P} & \mathbf{S} & \mathbf{0} & \mathbf{T} & \mathbf{P} & \mathbf{S} & \mathbf{P} & \mathbf{P} & \mathbf{P} & \mathbf{M} & \mathbf{A} & \mathbf{T} & \mathbf{V} & \mathbf{D} & \mathbf{P} & \mathbf{A} & \mathbf{S} & \mathbf{P} & \mathbf{A} & \mathbf{P} & \mathbf{A}\end{array}$ 991 tcaatagagcccctgccetgt ccccatccattgttgttaactccaccetct catcct atgtggcaaaccaggcatcttctggagctggg $\begin{array}{lllllllllllllllllllllllllllllll}331 & S & I & \mathbf{E} & \mathbf{P} & \mathbf{P} & \mathbf{A} & \mathbf{L} & \mathbf{S} & \mathbf{P} & \mathbf{S} & \mathbf{I} & \mathbf{V} & \mathbf{V} & \mathbf{H} & \mathbf{S} & \mathbf{T} & \mathbf{L} & \mathbf{S} & \mathbf{S} & \mathbf{Y} & \mathbf{V} & \mathbf{A} & \mathbf{H} & \mathbf{0} & \mathbf{A} & \mathbf{S} & \mathbf{S} & \mathbf{G} & \mathbf{A} & \mathbf{G}\end{array}$ 1081 ggtcagcccaatatcaccaagt tgattattaccaaacaaatgttgccetcttctattactatgtctcaaggagggatggttactgttatc $\begin{array}{lllllllllllllllllllllllllllllllll}361 & G & \mathbf{0} & \mathbf{P} & \mathbf{H} & \mathbf{I} & \mathbf{T} & \mathbf{K} & \mathbf{L} & \mathbf{I} & \mathbf{I} & \mathbf{T} & \mathbf{K} & \mathbf{0} & \mathbf{M} & \mathbf{L} & \mathbf{P} & \mathbf{S} & \mathbf{S} & \mathbf{I} & \mathbf{T} & \mathbf{M} & \mathbf{S} & \mathbf{0} & \mathbf{G} & \mathbf{G} & \mathbf{M} & \mathbf{V} & \mathbf{T} & \mathbf{V} & \mathbf{I}\end{array}$ 1171 ccagccacagtggtgacctcccgagggc tccaact aggcc aaaccagtacagctactatccagcccagtc aacaagcccagattgtcact

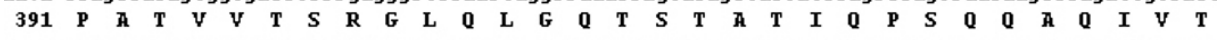

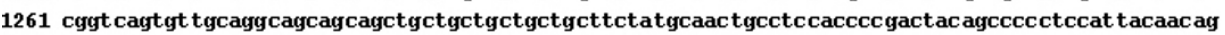
$\begin{array}{llllllllllllllllllllllllllllllll}421 & \mathbf{R} & \mathbf{S} & \mathbf{V} & \mathbf{L} & \mathbf{0} & \mathbf{A} & \mathbf{A} & \mathbf{A} & \mathbf{A} & \mathbf{A} & \mathbf{A} & \mathbf{A} & \mathbf{A} & \mathbf{A} & \mathbf{S} & \mathbf{M} & \mathbf{0} & \mathbf{L} & \mathbf{P} & \mathbf{P} & \mathbf{P} & \mathbf{R} & \mathbf{L} & \mathbf{0} & \mathbf{P} & \mathbf{P} & \mathbf{P} & \mathbf{L} & \mathbf{0} & \mathbf{0}\end{array}$ 1351 atgccacagccccegactcagc agcaagttaccattctgc agcagcetcctccactcc aggccatgcaac agcetccacctc agaaagtt

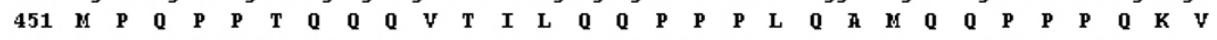
1441 cgaatcaatt tacagcaacagcetcctcctctgcagatcaagagtgtgcetctacccactttgaaaatge agactaccttagtcccacca

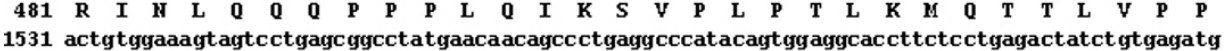

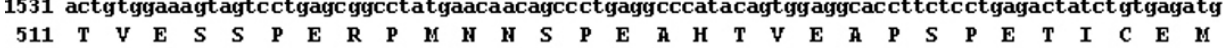
1621 atcacagatgtagttcctgaggtt tgagt ctccttctcagatggatgrt gaat tggtgagt gggt ctcctgtggeactctcacccagcct $\begin{array}{llllllllllllllllllllllllllllllll}541 & \text { I } & \text { T } & \text { D } & \text { V } & \text { V } & \text { P } & \text { E } & \text { V } & \text { E } & \text { S } & \text { P } & \text { S } & \mathbf{Q} & \text { M } & \text { D } & \text { V } & \text { E } & \text { L } & \text { V } & \text { S } & \text { G } & \text { S } & \text { P } & \text { V } & \text { A } & \text { L } & \text { S } & \text { P } & \mathbf{Q} & \mathbf{P}\end{array}$ 1711 cgatgtgtgaggtctggttgtgagaacctcccattgtgagtaaggactgggacaatgaat act gcagcaatgagt gtgtggtgaagc ac $\begin{array}{lllllllllllllllllllllllllllllllllll}571 & \text { R } & \text { C } & \text { V } & \text { R } & \text { S } & \text { G } & \text { C } & \text { E } & \text { H } & \text { P } & \text { P } & \text { I } & \text { V } & \text { S } & \text { K } & \text { D } & \text { W } & \text { D } & \text { H } & \text { E } & \text { Y } & \text { C } & \text { S } & \text { H } & \text { E } & \text { C } & \text { V } & \text { V } & \text { K } & \text { H }\end{array}$ 1801 tgcagggatgtattct tggcct gggtagcctctagaaat caaacacagtggtgtttgtgaaat ag

$\begin{array}{llllllllllllllllllllllll}601 & C & R & D & \text { Y } & \text { F } & \text { L } & \text { A } & \text { W } & \text { Y } & \text { A } & \text { S } & \text { R } & \text { H } & \text { S } & \text { H } & \text { T } & \text { Y } & \text { Y } & \text { F } & \text { Y } & \text { K } & \text { * }\end{array}$
}

Figure 2. Nucleotide and deduced amino acid sequences of KIAA0737 fragments that interact with PNUTS (1-433) in the yeast two-hybrid system. The full-length KIAA0737 consist of 1866 nucleotides encoding a protein of 621 amino acids. The dotted box indicates the putative NLS (nuclear localization signal) sequence at residue 199-218. Residue 223275 is a HMG-box (gray box). 
consists of 1866 nucleotides, encoding a protein of 621 amino acids (Figure 2). The predicted molecular mass of the protein is $68.3 \mathrm{kDa}$. Putative conserved domains, such as a HMG (high-mobility group)-box sequence (at residue 199-218) and a NLS (at residue 223-275), were detected (Figure 2) in the BLAST search.

\section{Characterization of the interaction between PNUTS and LCP1}

Co-immunoprecipitation experiments were performed to verify the interaction between PNUTS and LCP1. Plasmids encoding PNUTS and LCP1 fused to either an amino-terminal FLAG or His- tag were transiently co-transfected into 293T cells. Immunoprecipitation of FLAG-PNUTS resulted in co-precipitation His-LCP1 (Figure 3A). Conversely, HisPNUTS was co-precipitated by FLAG-LCP1 (Figure 3B). These results suggest that PNUTS physically associates with LCP1 in mammalian cells.

We further examined the interaction between PNUTS and LCP1 by assessing the binding affinities of various fragments of the two interacting proteins. Since even small fragments of LCP1 (LCP1 538-621) were able to interact with PNUTS in the yeast two hybrid assay (clone \#28 in Table 1), plasmids encoding the corresponding Histagged fragments of LCP1 (Figure 4A) were constructed and used to identify the PNUTS-binding domain of LCP1. Each His-tagged fragment of LCP1 was co-transfected with full length FLAGtagged PNUTS in 293T cells and the cell lysates were then subjected to immunoprecipitation with an anti-FLAG affinity resin (Figure 4A). In Western blots with anti-His antibody, LCP1 (full length) and LCP1 (415-621) were found to bind to full length PNUTS, but no binding was detected for LCP1

A

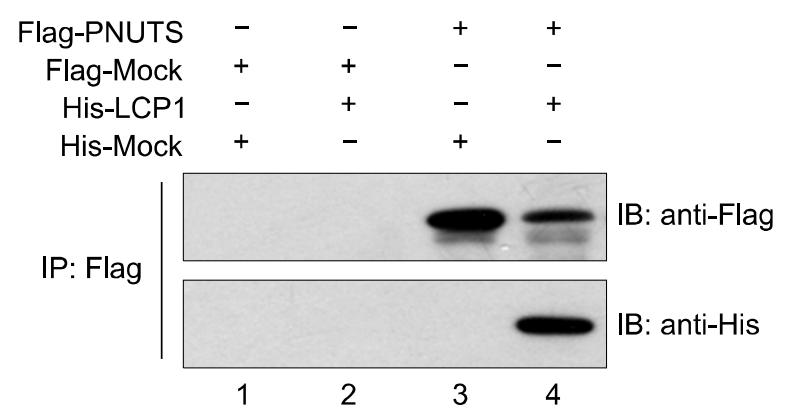

(1-537), LCP1 (415-591), or LCP1 (415-537) (Figure $4 \mathrm{~B})$. These results suggest that the PNUTS-binding domain is close to the C-terminus of LCP1 (592-621).

Similar studies employing various Flag-tagged PNUTS fragments were carried out to identify the LCP1-binding domain of PNUTS. PNUTS (full length), PNUTS (309-872), PNUTS (590-872), PNUTS (143-433), and PNUTS (1-263) (Figure 4C) were transiently co-transfected with His-tagged full length LCP1 into 293T cells and the cell lysates were subjected to immunoprecipitation with antiFLAG antibody resin. As shown in Figure 4D, PNUTS (full length) and PNUTS (1-263) were able to co-precipitate LCP1, but the other PNUTS fragments, including PNUTS (143-433), failed to bind LCP1. These results indicate that an N-terminal region of PNUTS distinct from the PP1-binding region located in residues (398-401) interacts with LCP1. In summary, we found that an N-terminal region of PNUTS binds to the C-terminus of LCP1 (Figure 4E).

\section{Nuclear co-localization of PNUTS and LCP1}

PNUTS localizes to the nucleus during interphase (Allen et al., 1998). LCP1 has been classified as a member of the TOX family of proteins, which also localizes to the nucleus (O'Flaherty and Kaye, 2003). To confirm that PNUTS and LCP1 co-localize in the nucleus, we cloned PNUTS and LCP1 cDNA into a green and red fluorescence protein expression vector, respectively, and co-transfected them into HeLa cells. By confocal microscopy, both the green fluorescence of the GFP-PNUTS fusion protein and the red fluorescence of the RFP-LCP1 fusion protein were observed in the nuclei (Figure $5 A)$. At higher resolution, the fluorescent-tagged

B

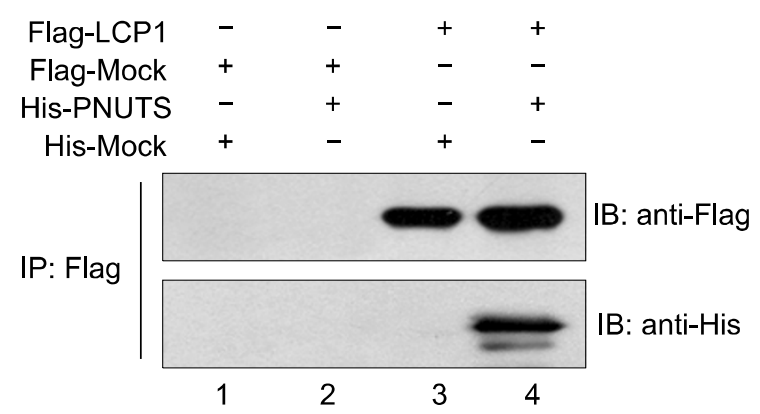

Figure 3. LCP1 interacts with PNUTS in HEK 293T cells. (A) His-tagged LCP1 co-immunoprecipitates with FLAG-tagged PNUTS. Immunoblot analysis of proteins prepared from 293T cells transfected with LCP1, and PNUTS expression constructs or with expression vector alone. Anti-FLAG immunoprecipitate blots were probed with anti-FLAG antibody (top) and then stripped and re-probed with anti-his antibody (bottom). His-tagged LCP1 was detected only in lane 4. (B) His-tagged PNUTS co-immunoprecipitates with Flag-tagged LCP1. The blot was probed sequentially with anti-Flag antibody (top) and anti-His antibody (bottom). His-tagged PNUTS was also detected in lane 4. 
A

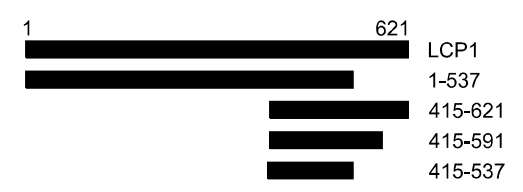

B

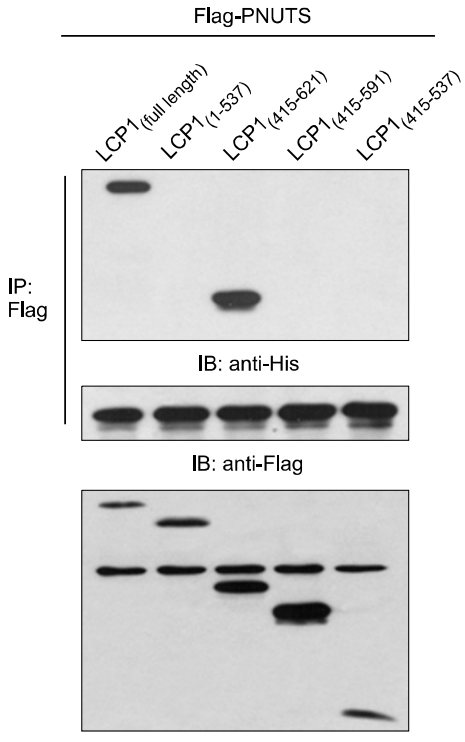

IB: anti-His
C

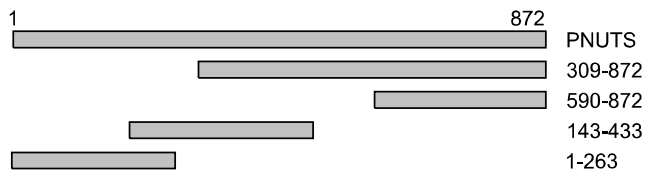

D

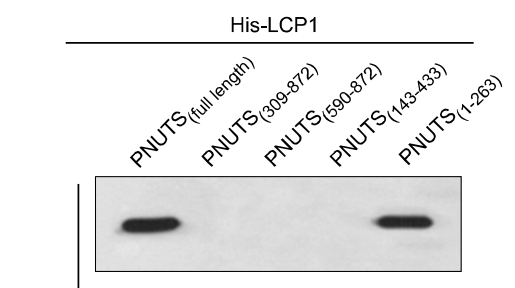

IB: anti-His

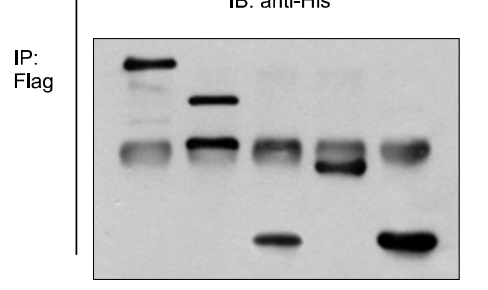

IB: anti-Flag

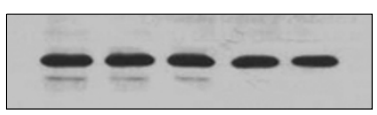

IB: anti-His

E

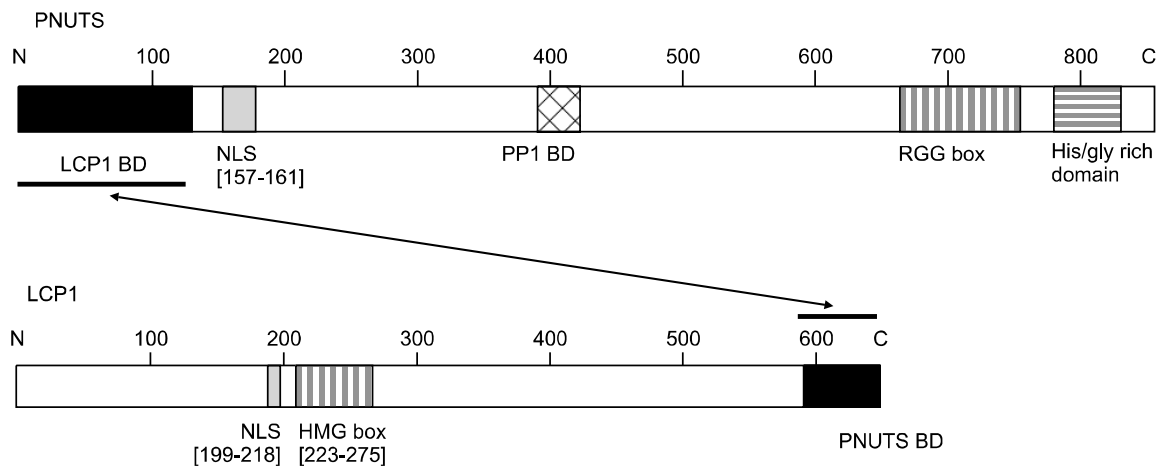

Figure 4. Identification of interaction domains. (A) Schematic of His-tagged fragments of LCP1. (B) His-tagged fragment of LCP1 co-immunoprecipitates with FLAG-tagged PNUTS. FLAG i.p., anti-FLAG immunoprecipitate blots were probed with anti-FLAG antibody and then stripped and re-probed with anti-his antibody. LCP1 (full length) and LCP1 (415-621) were found to bind to full length PNUTS. Some of the cell lysates were immunoblotted with anti-his antibody (bottom) prior to the Flag i.p.. (C) Schematic of Flag-tagged fragments of PNUTS. (D) Flag-tagged fragment of PNUTS co-immunoprecipitates with His-tagged LCP1 (full length). FLAG i.p., anti-FLAG immunoprecipitate blots were probed with anti-His antibody and then stripped. Some of the cell lysates were immunoblotted with anti-His antibody (bottom) prior to the FLAG i.p.. PNUTS (full length) and PNUTS (1-260) were found to bind to LCP1 (full length). After the FLAG i.p., some of the cell lysates were immunoblotted with anti-FLAG antibody. (E) Schematic of putative interaction region between PNUTS and LCP1 protein. proteins appear as speckles in nucleus. The nuclear speckles can be seen in the merged images of the GFP and RFP proteins, indicating that specific fractions of these proteins are co-localized in the nucleus (Figure 5A). Primary sequence analysis indicates that LCP1 has a putative nuclear localization signal (NLS) at residues 198-218. We examined the NLS of LCP1 using deletion constructs. As shown in Figure 5B, deletion of the putative NLS motif of LCP1 led to the cytosolic location of the protein, whereas the constructs that retain the NLS had apparent nuclear localizations. In particular, LCP1 (190-621) co-localized with PNUTS and formed nuclear speckles (Figure 5B).

\section{Transcriptional activity and transactivation domain of LCP1}

HMG-box family proteins are found in a variety of eukaryotic organisms and most are known or suspected regulators of gene expression (Wang et al., 1999; Scaffidi et al., 2002). Since LCP1 is a nuclear protein that contains a HMG-box motif, we tested it for potential transcriptional activity. We used a GAL4-based heterologous luciferase reporter system (Figure 6A) because target genes of LCP1 are not known at this time. A 5X-GAL4-Luc firefly 


\section{A}

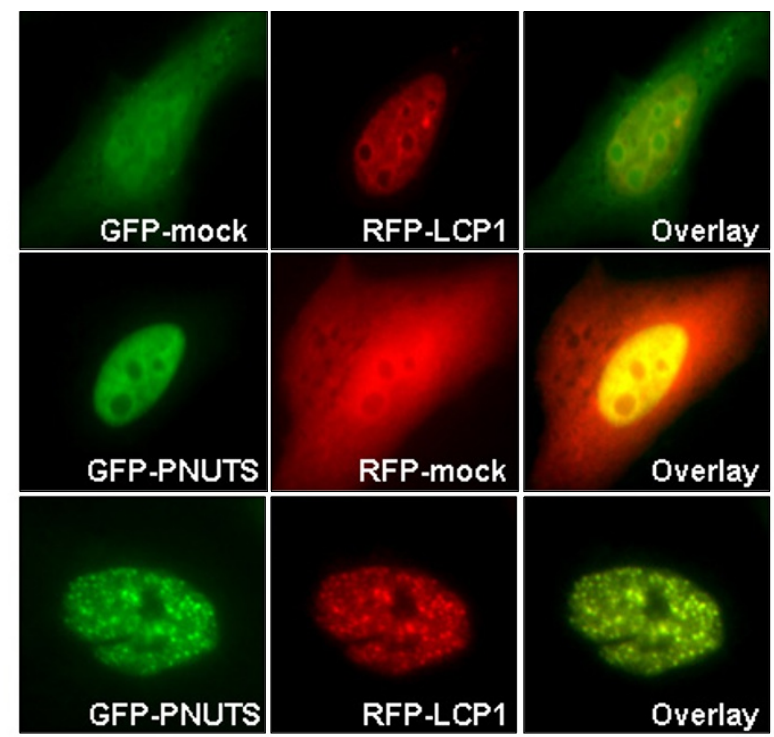

B

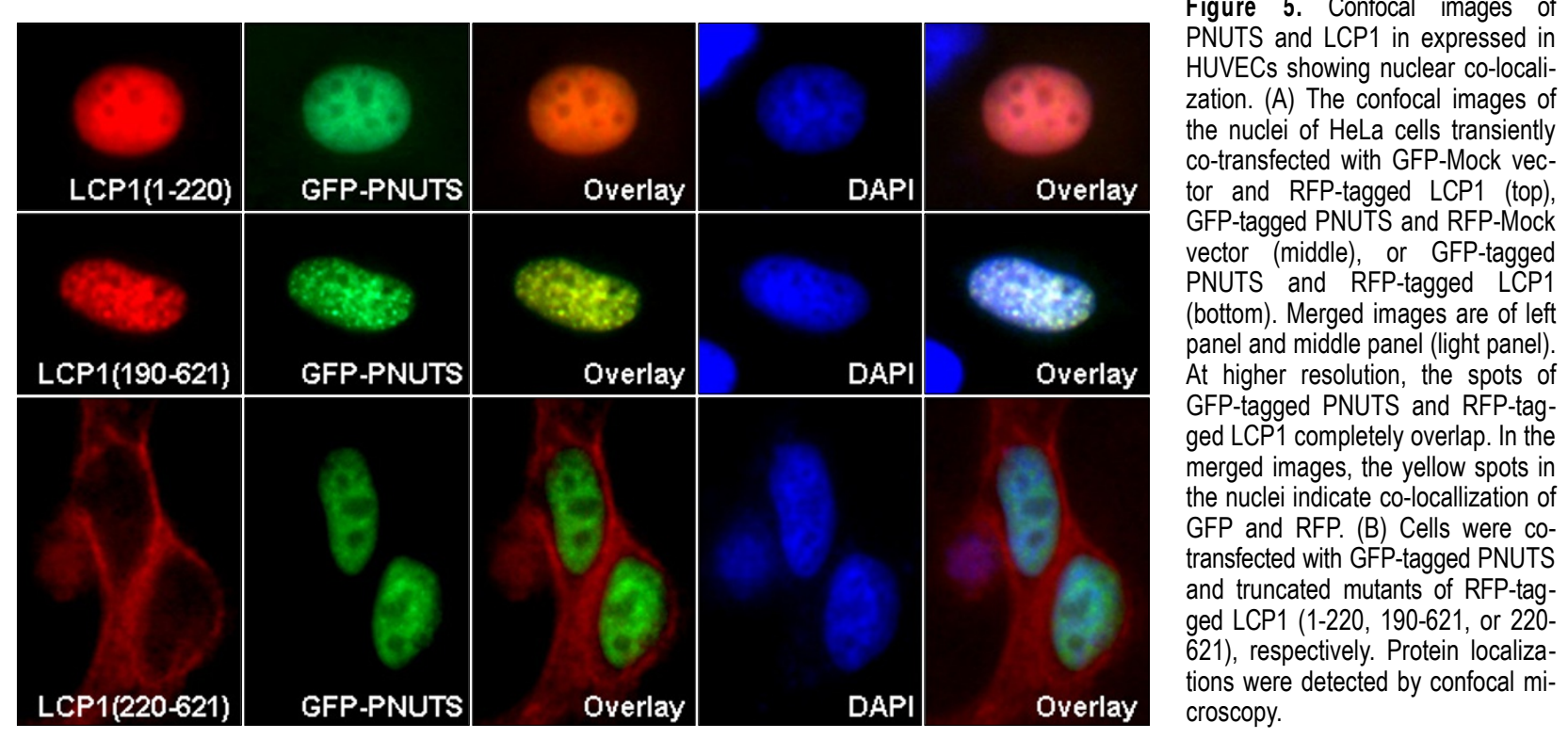

luciferase reporter plasmid and a plasmid encoding the GAL4 DNA-binding domain fused to the LCP1 protein (GBD-LCP1) were co-transfected into HEK 293 cells. The expression of the GBD-LCP1 fusion protein enhanced the GAL4 reporter luciferase activity 2-3 fold, whereas GBD-PNUTS failed to activate luciferase expression, suggesting that LCP1 is a transcriptional activator (Figure 6B).

We then delineated the putative trans-activation domain of LCP1 using various deletion constructs of GBD-LCP1 (Figure 6C). All constructs lacking the N-terminal 200 amino acids of LCP1 were unable to induce the GAL4-driven luciferase expre- ssion (Figure 6D). In contrast, GBD-LCP1 constructs containing the first 220 amino acids of the $N$ terminus had very strong transcriptional activities (Figure 6D). These results indicate that LCP1 possesses a trans-activation domain in its $\mathrm{N}$ terminus. Notably, the inclusions of the HMG-box motif in the N-terminal constructs of GBD-LCP1 resulted in a suppression of the transcriptional activity of LCP1 (Figure 6D). This suggests that the HMG-box motif in GBD-LCP1 may hamper the proper binding of GDB to the GAL4 binding site, presumably because the protein is titrated to its endogenous HMG-box binding sites on the chro- 
A

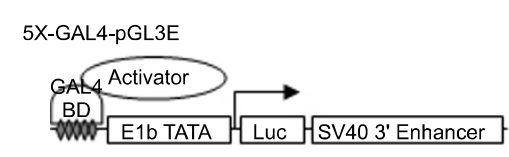

0.01:5X GAL4 binding site (UAS)

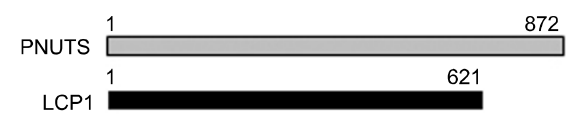

B

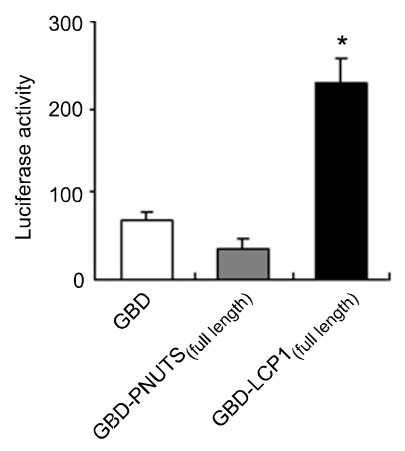

C

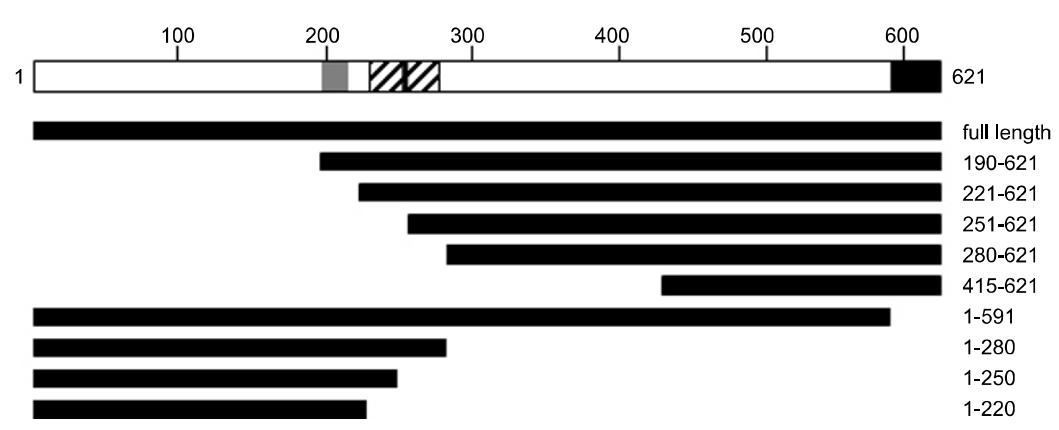

D

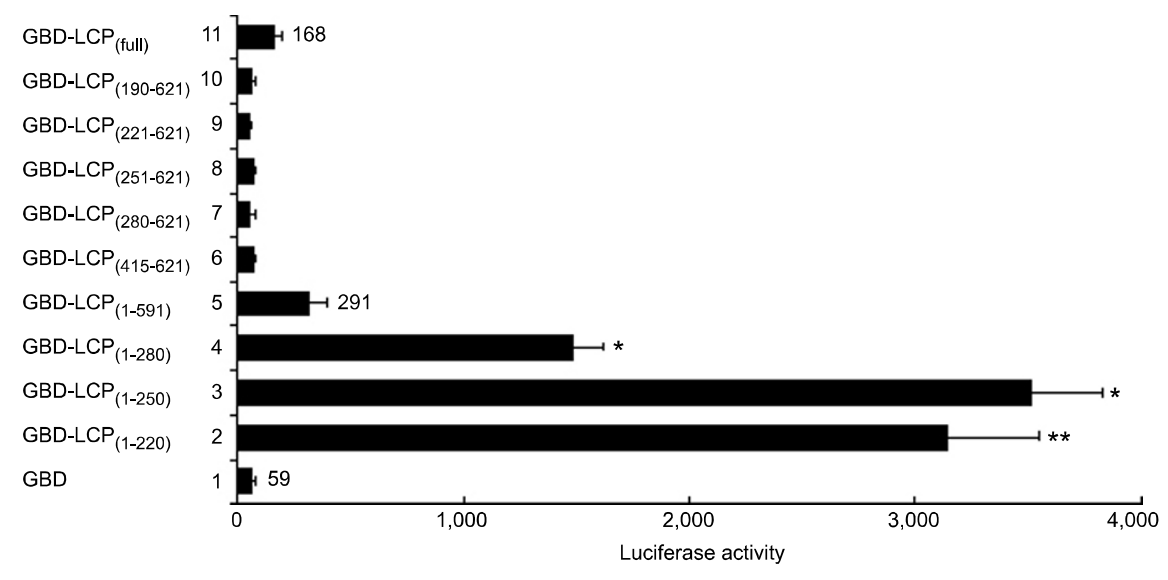

Figure 6. Identification of the transactivation domain of LCP1. (A) Schematic representation of the GAL4 reporter assay (upper panel) and GBD fusion region of PNUTS and LCP1 (lower panel); (B) Transcriptional activities of PNUTS and LCP1 $\left({ }^{*} P<\right.$ 0.001 vs. GBD); (C) Domain mapping of various LCP1 deletion mutants; (D) Identification of the transactivation domain of LCP1 in HEK 293 cells: HEK 293 cells were co-transfected with the 5X-GAL4pGL3E reporter construct along with expression vehicles encoding GBD, GBD-LCP1 full length, or GBDLCP1 deletion mutants, and luciferase activity was subsequently measured. Similar results were obtained from three independent experiments. Graph represents mean values \pm SEM. $\left({ }^{*} P<0.001\right.$ and ${ }^{* *} P<0.05$ vs. GBD).

mosomal DNA.

\section{Inhibition of the transcriptional activity of LCP1 by PNUTS}

To determine whether PNUTS modulates the transcriptional activity of LCP1, we constructed GBDLCP1 (full: $\triangle 221-499)$ and GBD-LCP1 (1-591: 221 499); two proteins that differ in their PNUTSbinding affinities (Figure 7A). GBD-LCP1 (1-591: $\triangle 221-499)$, which lacks the PNUTS binding motif, has a much strong transcriptional activity than GBDLCP1 (full:D221-499), which contains the PNUTS binding motif (Figure 7B). Co-transfection of PNUTS significantly reduced the transcriptional activity of GBD-LCP1 (full: $2221-499$ ) in the luciferase reporter assay, but had no significant effect on the activity of GBD-LCP1 (1-591: $221-499)$ (Figure 7B). Conversely, co-transfection of PNUTS (1-263), which blocks interaction between LCP1 and full length PNUTS, or knockdown of endogenous PNUTS by siRNA significantly increased the activity of GBDLCP1 (full:A221-499) in the luciferase reporter assay (Figure 7B and C). These results indicate that PNUTS negatively regulates the transcriptional activity of LCP1 through direct binding.

Since PNUTS forms a complex with PP1, we tested whether PP1 activity or the interaction of 
A

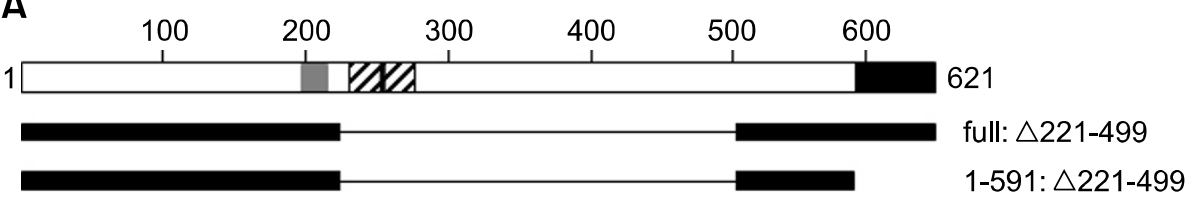

B

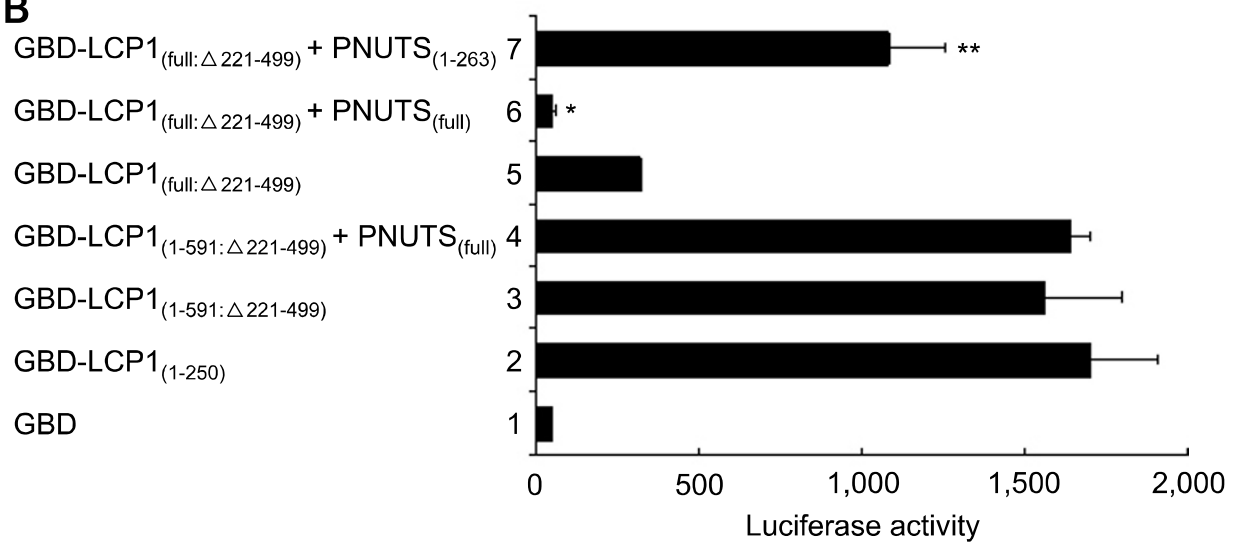

C

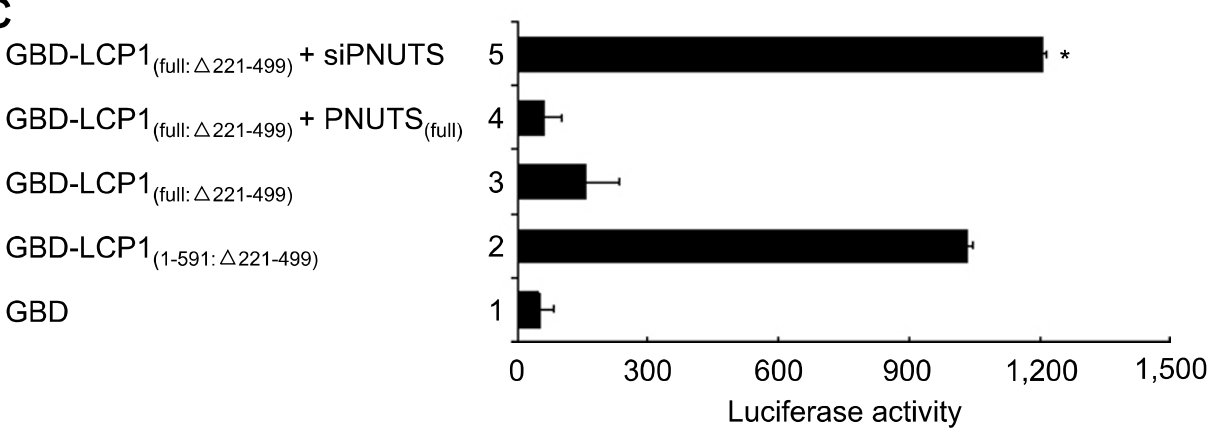

Figure 7. PNUTS can repress the transcriptional activity of LCP1. (A) Diagram of LCP1 deletion mutants; (B) HEK 293 cells were co-transfected with expression vectors for PNUTS (full length), PNUTS (1-263) mutants, GBD-LCP1 (full: $\triangle 221-499$ ), and/or GBD-LCP1 (1-591: $\triangle 221-499)$ together with the response promoter for GAL4 (5X-GAL4-pGL3E). The GBD-LCP1 (1-250) construct was used as the positive control of transcriptional activity ${ }^{*} P<0.001$ and ${ }^{* *} P<0.05$ vs. GBD-LCP1 (full: $\triangle 221-$ 499)). (C) Cells were co-transfected with siRNA constructs for PNUTS (siPNUTS), expression vectors for PNUTS (full length), and/or GBDLCP1 (full: $\triangle 221-499$ ), or GBD-LCP1 (1-591: $\triangle 221-499)$ together with $5 X$ GAL4-pGL3E ( ${ }^{*} P<0.001$ vs. GBDLCP1 (full: $\Delta 221-499)$ ).

PNUTS with PP1 is involved in regulating the transcriptional activity of LCP1. Okadaic acid, an inhibitor of PP1, does not affect the transcriptional activities of either GBD-LCP1 (full: $\Delta 221-499$ ) or GBD-LCP1 (1-591: $\triangle 221-499)$ in the luciferase assay (Figure 8A). Moreover, co-transfection of PNUTS (357-433) or PNUTS (357-486), which block the binding of PNUTS to PP1, had no significant effect on the activity of GBD-LCP1 (full: $\triangle 221-499$ ) (Figure $8 \mathrm{~B}$ ). These results indicate that PP1 is not required for the inhibitory effect of PNUTS on the transcriptional activity of LCP1.

\section{Discussion}

In the present study, we report that LCP1, a member of the TOX HMG-box subfamily, is a novel binding partner of PNUTS in mammalian cells. The yeast two-hybrid assay and biochemical analysis have revealed that LCP1 tightly associates with PNUTS through its C-terminus. In turn, PNUTS interacts with LCP1 through a region in its $\mathrm{N}$ terminus, which is distinct from its PP1 binding motif (Allen et al., 1998). These results indicate that LCP1 may form ternary complexes with PNUTS and PP1 in certain cellular contexts.

Immunofluorescence studies show that a subpopulation of LCP1 co-localizes with PNUTS in the nucleus and often forms nuclear speckles. Notably, the TOX family members are known to contain a conserved multiple lysine-based NLS sequences

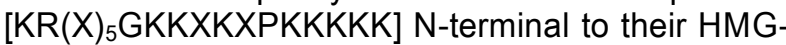
box motifs (O'Flaherty and Kaye, 2003). LCP1 possesses a similar putative NLS, except that it lacks the second basic residue of the consensus motif. Deletion of this putative NLS resulted in complete loss of nuclear localization of LCP1. These data confirm that LCP1 is localized in the nucleus through an NLS that has a similar architecture as other TOX family NLS domains.

Sequence analysis revealed that LCP1 encodes 

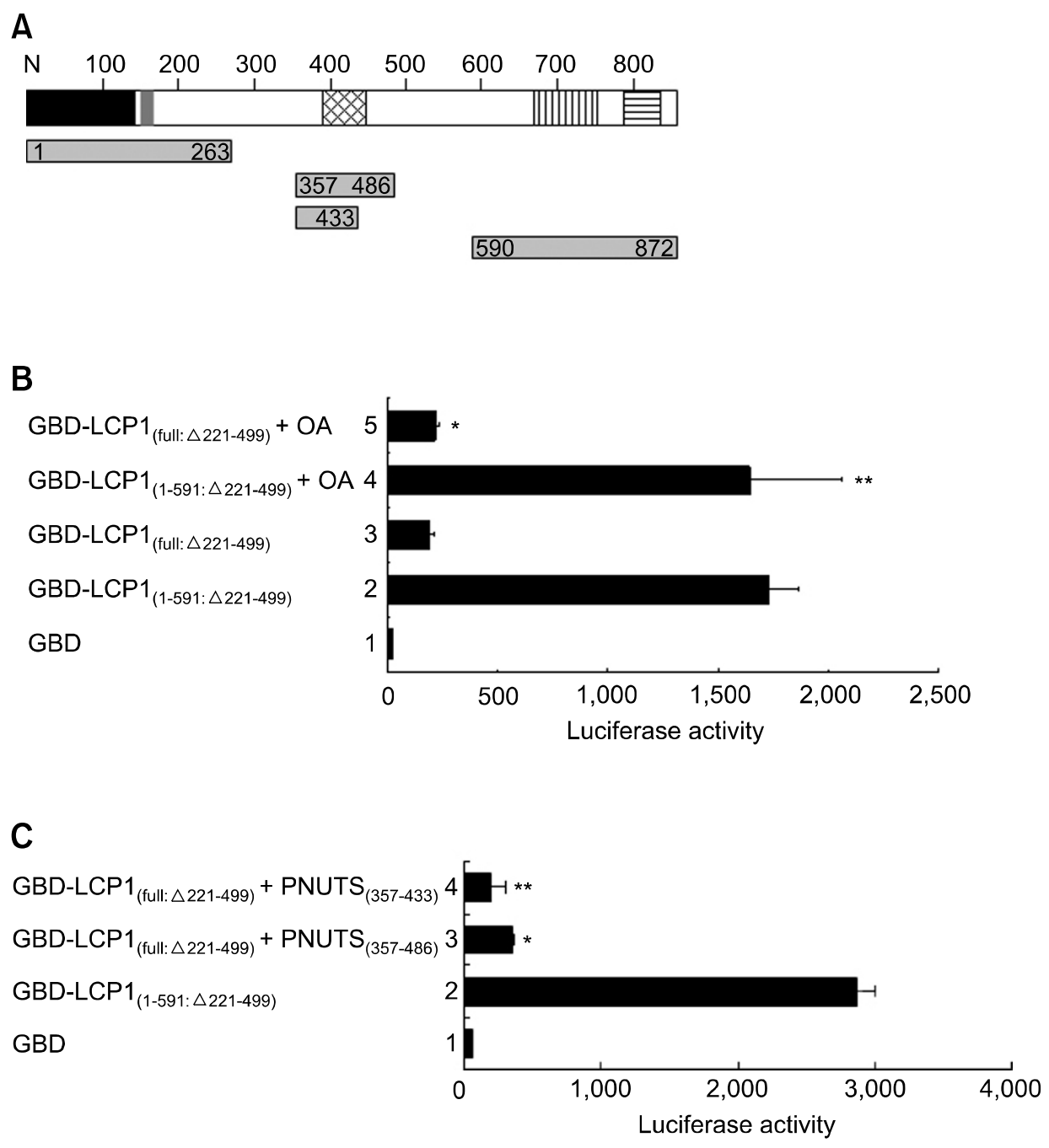

Figure 8 Regulation of LCP1 transcriptional activity by PNUTS. (A) The domain organization of PNUTS; (B) HEK 293 cells were co-transfected with GBD (empty vector), GBD-LCP1 (full: $\triangle 221-499$ ), or GBDLCP1 (1-591: $\triangle 221-499)$ together with 5X-GAL4-pGL3E for $16 \mathrm{~h}$ and then treated with $100 \mathrm{nM}$ okadaic acid (OA) for $4 \mathrm{~h}\left({ }^{*} P<0.001\right.$ vs. GBDLCP1 (full: $\triangle 221-499),{ }^{* *} P<0.05$ vs. GBD-LCP1 (1-591: $\triangle 221-499))$. (C) Cells were co-transfected with expression vectors for PNUTS (full length), various PNUTS mutants (W401A, 357-537, 357-486, 357-433, and 590-872), GBD-LCP1 (full: $\triangle 221$ 499), and/or GBD-LCP1 (1-591: $\triangle 221-499)$ together with 5X-GAL4pGL3E $\left({ }^{*} P<0.001\right.$ and ${ }^{* *} P<$ 0.05 vs. GBD-LCP1 (full: $\triangle 221-499)$ ).

a 70-80 amino acid DNA-binding domain (the HMG-box), which is quite conserved in all members of the HMG-box family and related to a motif originally identified in HMGB1 (Baxevanis and Landsman 1995; O'Flaherty and Kaye, 2003). This motif appears to be involved in both protein-protein interactions and the recognition of DNA (Aidinis et al., 1999; O'Flaherty and Kaye, 2003). It has been reported that RAG1 interacts with the tandem HMG-boxes of HMGB1 or HMGB2 (Aidinis et al., 1999; O'Flaherty and Kaye, 2003). Alternatively, the HMG-box interacts with the minor groove of the DNA helix (Read et al., 1993; Bewley et al., 1998) and thus, HMG-box family proteins are considered to be regulators of gene expression. Indeed, the TOX protein, which resembles LCP1 with its bipartite NLS sequence and single centrally located HMG-box motif, has been shown to be involved in the regulation of gene expression during thymocyte selection and development of CD4 T cells (Wilkinson et al., 2002; Aliahmad and Kaye, 2008). In this study, we employed a GAL4-based heterologous luciferase reporter system to show that LCP1 has transcriptional activity. Interestingly, the $\mathrm{N}$-terminus of LCP1 has a strong trans-activation activity, whereas the C-terminal domain, which is crucial for PNUTS binding, lacks this activity. Notably, LCP1 N-terminal fragments that include the HMG-box motif have much lower trans-activation activity in the GAL4 reporter system than their HMG-box deleted counterparts, indicating that the HMG-box of LCP1 binds to endogenous sites in the chromosomal DNA. Sequence analysis has suggested that the HMG-box sequence of LCP1 resembles those of sequenceindependent DNA binding proteins of the HMG-box family (O'Flaherty and Kaye, 2003). On the other hand, the N-terminal domain of LCP1 is not homologous to the amino acid sequences of any known protein domains. However, this domain appears to be acidic and more similar to other TOX subfamily 
members than the C-terminal region (O'Flaherty and Kaye, 2003). Considering that acidic domains are often involved in protein-protein interactions in the nucleus (O'Flaherty and Kaye, 2003; Cleynen and Van de Ven, 2008), our data imply that the $\mathrm{N}$-terminus of LCP1 may play a role in recruiting or setting up the transcriptional machinery at the chromosomal sites that are recognized by the HMG-box motif of LCP1.

This study also suggests a potential role for PNUTS in regulating LCP1-mediated transcriptional activation. Biochemical studies have shown that LCP1 binds to PNUTS through its C-terminus, which is distinct from its trans-activation and DNA binding domains. Interestingly, the transcriptional activity of LCP1 was strongly suppressed by increasing the available pool of PNUTS for binding to GBD-LCP1. Conversely, knockdown of PNUTS in cells significantly increased LCP1-mediated transcription. In many cases, PP1 binding proteins have been shown to exert their functions through the regulation of PP1 targeting or catalytic activity (Cohen, 2002; Ito et al., 2004). However, the inhibitory effect of PNUTS toward LCP1 was not significantly affected by blocking PP1 activity or by the interaction of PNUTS with PP1. Therefore, it is more likely that PNUTS induces a conformational change in LCP1 via direct binding to the C-terminus of LCP1, which could result in impaired complex formation with the transcriptional machinery.

PNUTS is expressed in various tissues and most types of mammalian cells (Allen et al., 1998). Likewise, LCP1 mRNA is detected in most tissues, with relatively high expression levels in testis and skin (O'Flaherty and Kaye, 2003). PNUTS is induced by hypoxia and the levels of this protein in various cell types are correlated with p53 levels (Udho et al., 2002). It is likely that the expression levels of LCP1, as well as PNUTS, change during embryonic development and in response to cellular changes, including differentiation, apoptosis, and senescence. Therefore, it is probable that the spatial and temporal patterns of these proteins may specifically control the expression of genes that are critical for certain physiological and pathological processes. This possibility is currently under investigation.

\section{Methods}

\section{Yeast two-hybrid library screens}

Protein-protein interactions in the yeast two-hybrid screens were identified using the MATCHMAKER GAL4 TwoHybrid system, according to the manufacturer's protocol (Clontech Palo Alto, CA). We used the yeast strain Saccharomyces cerevisiae CG1945, included in the MATCHMAKER GAL4 Two-Hybrid System (Clontech). Yeast were grown in either YPD medium or, once transformed, were maintained in appropriate synthetic dropout (SD) medium to select for plasmids. The auxotrophic markers of the CG1945 strain were verified before transformation. Briefly, the strain was streaked on appropriate SD selection plates, SD/Trp-, SD/Leu-, SD/His-, SD/Ura-, YPD plates, and incubated at $30^{\circ} \mathrm{C}$ for 4-6 days to allow the phenotype to appear. The strain should not grow on SD/Trp-, SD/Leu-, or SD/His- plates, but should grow on SD/Ura-, YPD plates. CG1945 strain is slightly leaky for HIS3 expression due to the presence of a minimal HIS3 promoter upstream of the gene. Therefore, we determined the optimum concentration of 3-amino- 1,2,4-triazole (3-AT), a competitive inhibitor of the yeast His3 protein, to suppress background growth on SD medium lacking histidine during the two- hybrid screen. A minimum concentration of $1 \mathrm{mM}$ 3-AT in the medium was sufficient to suppress background growth of the untransformed CG1945 strain and the transformants.

The bait plasmid DNA, the GAL4 DNA-binding domain (GBD)/PNUTS (1-433) in pAS2-1 (Clontech) with the TRP1 selectable marker, was constructed and named pAS2-1/PNUTS (1-433). DNA fragments of rat PNUTS (1-433) were amplified by Pfu polymerase. PCR products were digested by EcoRI and $\mathrm{BamHI}$ and subcloned into the pAS2-1 vector (Clontech). The Human Brain cDNAs fused with the GAL4-activation domain (GAL4AD) were used as prey plasmids (Clontech) to generate the two-hybrid cDNA library. $L E U 2$ is the selectable marker in prey vector.

The CG1945 cells were transformed with the GBDPNUTS (1-433) bait plasmid using the lithium acetate method (clontech). GAL4AD vector or the human brain cDNA library was transformed into the transformants carrying the GBD-PNUTS (1-433) bait plasmid with the following large scale transformation procedure: Freshly grown transformants carrying the GBD-PNUTS (1-433) bait plasmid were transferred to $1 \mathrm{~L}$ of fresh SD/Trp-medium and grown until the $\mathrm{OD}_{600}$ reached 0.5 . The cells were harvested, washed in $500 \mathrm{ml}$ of sterile TE $(10 \mathrm{mM}$ Tris- $\mathrm{HCl}$, $\mathrm{pH} 7.5,1 \mathrm{mM}$ EDTA), and resuspended in $8 \mathrm{ml}$ of $1 \times$ TE/LiAc (TE with $100 \mathrm{mM}$ lithium acetate). This $8 \mathrm{ml}$ of competent cell suspension was added to a tube containing $0.5 \mathrm{mg}$ of the library cDNA and $20 \mathrm{mg}$ of the denatured salmon sperm carrier DNA, mixed well by vortexing, and combined with $60 \mathrm{ml}$ of fresh PEG/LiAc solution (50\% PEG in TE/LiAc solution). Immediately after mixing by high speed vortexing, the transformation mixtures were incubated at $30^{\circ} \mathrm{C}$ for $30 \mathrm{~min}$ with shaking $(200 \mathrm{rpm})$. Then, 7 $\mathrm{ml}$ of DMSO was added to the incubated mixtures, which were then heat-shocked at $42^{\circ} \mathrm{C}$ for 15 min with occasional swirling. The mixtures were immediately chilled on ice for $2 \mathrm{~min}$, centrifuged, and then resuspended in $10 \mathrm{ml}$ of $1 \times$ TE. These resuspended cells were then plated on SD lacking tryptophan, leucine, and histidine but containing $1 \mathrm{mM} 3-\mathrm{AT}$, and incubated for 8 days at $30^{\circ} \mathrm{C}$. The rapidly growing pink colonies were isolated as positive colonies for further analyses. After the positive colonies were verified for real protein-protein interactions using the colony-lift filter $\beta$-galactosidase assay, as described below, the yeast plasmid DNA from each positive clone was prepared. Briefly, the candidate clones were re-streaked on SD/Leucontaining $1 \mu \mathrm{g} / \mathrm{ml}$ cycloheximide to eliminate the bait GBD-PNUTS (1-433) fusion DNA. The GBD-PNUTS (1-433) 
fusion constructs in pAS2-1 carry the CYHs2 gene for cycloheximide sensitivity, so the selected colonies grown on SD/Leu-containing $1 \mu \mathrm{g} / \mathrm{ml}$ cycloheximide carried only prey plasmids. The yeast DNAs from these colonies were then isolated as follows: $0.5 \mathrm{ml}$ of fresh liquid culture was harvested, resuspended in $50 \mu \mathrm{l}$ of the residual liquid and treated with $10 \mu \mathrm{l}$ of lyticase, which weakens the tough cell wall, for $60 \mathrm{~min}$ at $37^{\circ} \mathrm{C}$ with shaking at $250 \mathrm{rpm}$, followed by addition of $10 \mu \mathrm{l}$ of $20 \%$ SDS with vigorous vortexing for $1 \mathrm{~min}$. The mixture was put through one freeze/thaw cycle (at $-20^{\circ} \mathrm{C}$ ) and lysed completely by vortexing. The volume of the lysate was brought to $200 \mu$ l with TE buffer ( $\mathrm{pH} 7.0$ ), and extracted with $200 \mu \mathrm{l}$ of phenol: chloroform: isoamylalcohol (25: 24: 1). The plasmid DNA in the aqueous phase was separated by centrifugation, precipitated by addition of $8 \mu \mathrm{l}$ of $10 \mathrm{M}$ ammonium acetate and $500 \mu \mathrm{l}$ of $100 \%$ ethanol, followed by incubation at $-70^{\circ} \mathrm{C}$ for $1 \mathrm{~h}$ and centrifugation at $14,000 \mathrm{rpm}$ for $10 \mathrm{~min}$. The crude yeast plasmid DNA was then transformed into competent $\mathrm{DH} 5 \alpha$ E.coli cells. The isolated plasmids were analyzed by Bg/ll restriction enzyme digestion to separate the human brain cDNA insert from GAL4AD/cDNA library vector. The DNAs were further analyzed by sequencing.

\section{Cell culture and cell lines}

HEK293 and HeLa cells were cultured in DMEM (GibcoBRL, Carlsbad, CA) supplemented with $10 \%$ FBS (BIO WHITTAKER), $100 \mathrm{units} / \mathrm{ml}$ penicillin, and $100 \mu \mathrm{g} / \mathrm{ml}$ streptomycin (Gibco- BRL) in a humidified atmosphere at $37^{\circ} \mathrm{C}$ with $5 \% \mathrm{CO}_{2}$.

\section{Preparation of mammalian expression plasmid}

PNUTS (full length), PNUTS (309-872), PNUTS (590-872), PNUTS (143-433), and PNUTS (1-263) were subcloned into pFLAG-CMV-2 expression vector (Sigma) by $5^{\prime}$ EcoRI and $3^{\prime}$ Notl sites. LCP1 (1-537), LCP1 (415-621), LCP1 (415-591), LCP1 (415-537), were subcloned into pcDNA3.1/ HisA expression vector (Invitrogen, Carlsbad, CA) by $5^{\prime}$ EcoRI and $3^{\prime}$ Xhol sites. For confocal microscopy, the open reading frame of LCP1 was cloned into red fluorescent vector, pDsRed-Express-C1 (clontech), and PNUTS was cloned into green fluorescent vector, pEGFP (clontech). Various expression vectors were amplified in $E$. coli XL1-blue and the plasmids were purified using QIAGEN Plasmid Maxi Kit.

\section{Transfection and transactivation assay}

HEK293 cells were seeded at $3 \times 10^{5}$ cells/well in 6 well plates. The GBD-fused LCP1 or PNUTS constructs were then co-transfected with GAL4-responsive luciferase reporter plasmid (5X-GAL4-pGL3E) using the Lipofectamine reagent (Invitrogen). Twenty four hours after transfection, cells were lysed in lysis buffer $(100 \mathrm{mM}$ potassium phosphate, $\mathrm{pH} 7.4,1 \%$ Triton X-100, $1 \mathrm{mM}$ DTT, and 20 mM EDTA). Cell debris was removed by centrifugation and 10-20 $\mu \mathrm{g}$ of total protein was used to measure the luciferase activity using the Luciferase Reporter Assay System (Promega, Madison, WI) and an automatic mi- croplate luminometer. The luciferase activity was routinely normalized to the amount of protein used in the assay. Luciferase activities were normalized with respect to parallel $\beta$-galactosidase activities, to correct for differences in transfection efficiency. Means were calculated from at least four independent experiments.

\section{Co-immunoprecipitation and Western blot analysis}

HEK 293T cells grown in DMEM (10\% FBS) were transiently co-transfected with various plasmids $(10 \mu \mathrm{g})$ using the calcium phosphate method. Cells were washed with fresh medium $5 \mathrm{~h}$ after transfection and were cultured for $16 \mathrm{~h}$. Cells were washed with PBS, harvested, and resuspended in lysis buffer (50 mM Tris-HCl, pH 7.4, $1 \mathrm{mM}$ EDTA, $1 \mathrm{mM}$ EGTA, $150 \mathrm{mM} \mathrm{NaCl}, 0.6 \mathrm{mM}$ PMSF, $20 \mu \mathrm{g} / \mathrm{ml}$ leupeptin and antipain, $10 \mu \mathrm{g} / \mathrm{ml}$ pepstatin $A$ and chymostatin, $0.5 \%$ Nonidet P-40). Cell lysates were briefly sonicated and centrifuged at $15,000 \mathrm{~g}$ for $20 \mathrm{~min}$. Protein concentrations of supernatants were determined using the BCA assay (Pierce, Rockford, IL). Cell lysates (1 mg) were incubated with anti-FLAG affinity beads (Sigma) for $2 \mathrm{~h}$ at $4^{\circ} \mathrm{C}$. Immuno-complexes were washed with lysis buffer and eluted from beads by boiling in SDS sample buffer. Samples were analyzed by SDS-PAGE $(12 \%$ polyacrylamide), proteins were transferred to PVDF membrane (Immobilon-P, Millipore) by electroblotting (200 mA, overnight). Blots were incubated with either an anti-FLAG antibody or an anti-His antibody (Santa Cruz, CA), followed by HRP-conjugated secondary antibody. Proteins were visualized using ECL.

\section{Confocal microscopy and fluorescence imaging}

To generate plasmids expressing PNUTS and LCP1, CDNA-fragments were amplified by PCR and cloned into pEGFP-C2 expression plasmid or pDsRed-express-C1 (Clontech, Palo Alto, CA). HeLa cells were cultured on glass-bottom dishes and transfected with pEGFP-C2PNUTS or pDsRed-express-C1-LCP1 using Lipofectamine reagent, according to the manufacturer's instructions (Invitrogen). After $24 \mathrm{~h}$, the cells were fixed with $2 \%$ paraformaldehyde and washed two or three times with PBS. Fluorescent images of EGFP and DsRed were obtained with an Olympus BX50EI confocal microscope controlled by Fluoview (Olympus, Tokyo), as described previously (Nagashima et al., 2002).

\section{Statistics}

Values of various parameters are presented as the mean \pm standard error of the mean (SEM). One-way analysis of variance (ANOVA) was used to compare each parameter. Post-hoc Student's $t$-test was also performed to identify which group difference accounted for the significant overall analysis of variance. A value of $P<0.05$ was considered statistically significant.

\section{Acknowledgments}

This work was supported by a grant from the 21C Frontier 
Functional Human Genome Project (FG08-21-15), a National Research Laboratory grant (ROA-2007-00020099-0(2008)), Ministry of Education, Science, and Technology (MEST), a grant from the Korea Health 21 R\&D Project, Ministry of Health Welfare and Family Affairs, Republic of Korea (A085136), and a Korean Research Foundation Grant funded by the Korean Government (KRF-2006-312-C00611).

\section{References}

Aidinis V, Bonaldi T, Beltrame M, Santagata S, Bianchi ME, Spanopoulou E. The RAG1 homeodomain recruits HMG1 and HMG2 to facilitate recombination signal sequence binding and to enhance the intrinsic DNA-bending activity of RAG1-RAG2. Mol Cell Biol 1999;19:6532-42

Aliahmad P, Kaye J. Development of all CD4 T lineages requires nuclear factor TOX. J Exp Med 2008;205:245-56

Allen PB, Kwon YG, Nairn AC, Greengard P. Isolation and characterization of PNUTS, a putative protein phosphatase 1 nuclear targeting subunit. J Biol Chem 1998;273:4089-95

Baxevanis AD, Landsman $D$. The HMG-1 box protein family: classification and functional relationships. Nucleic Acids Res 1995;23:1604-13

Bewley CA, Gronenborn AM, Clore GM. Minor groovebinding architectural proteins: structure, function, and DNA recognition. Annu Rev Biophys Biomol Struct 1998;27:10531

Cleynen I, Van de Ven WJ. The HMGA proteins: a myriad of functions (Review). Int J Oncol 2008;32:289-305

Cohen PT. Protein phosphatase 1--targeted in many directions. J Cell Sci 2002;115:241-56

De LG, Sherry TC, Krucher NA. Reduced Expression of PNUTS leads to activation of Rb-Phosphatase and Caspase-Mediated Apoptosis. Cancer Biol Ther 2008;7: 833-41

Depaoli-Roach AA, Park IK, Cerovsky V, Csortos C, Durbin SD, Kuntz MJ, Sitikov A, Tang PM, Verin A, Zolnierowicz S. Serine/threonine protein phosphatases in the control of cell function. Adv Enzyme Regul 1994;94:199-224

Graña X. Downregulation of the phosphatase nuclear targeting subunit (PNUTS) triggers pRB dephosphorylation and apoptosis in pRB positive tumor cell lines. Cancer Biol Ther 2008;7:842-4

Ito M, Nakano T, Erdodi F, Hartshorne DJ. Myosin phosphatase: structure, regulation and function. Mol Cell Biochem 2004;259:197-209

Kim YM, Watanabe T, Allen PB, Kim YM, Lee SJ, Greengard $P$, Nairn AC, Kwon YG. PNUTS, a protein phosphatase 1
(PP1) nuclear targeting subunit. Characterization of its PP1and RNA-binding domains and regulation by phosphorylation. J Biol Chem 2003;278:13819-28

Krucher NA, Rubin E, Tedesco VC, Roberts MH, Sherry TC, De Leon G. Dephosphorylation of Rb (Thr-821) in response to cell stress. Exp Cell Res 2006;312:2757-63

Landsverk HB, Kirkhus M, Bollen M, Küntziger T, Collas P. PNUTS enhances in vitro chromosome decondensation in a PP1-dependent manner. Biochem J 2005;390:709-17

Lee SJ, Lim CJ, Min JK, Lee JK, Kim YM, Lee JY, Won MH, Kwon YG. Protein phosphatase 1 nuclear targeting subunit is a hypoxia inducible gene: its role in post-translational modification of p53 and MDM2. Cell Death Differ 2007;6: 1106-16

Nagashima K, Endo A, Ogita H, Kawana A, Yamagishi A, Kitabatake A, Matsuda M, Mochizuki N. Adaptor protein Crk is required for ephrin-B1-induced membrane ruffling and focal complex assembly of human aortic endothelial cells. Mol Biol Cell 2002;13:4231-42

O'Flaherty E, Kaye J. TOX defines a conserved subfamily of HMG-box proteins. BMC Genomics 2003;4:13-22

Read CM, Cary PD, Crane-Robinson C, Driscoll PC, Norman DG. Solution structure of a DNA-binding domain from HMG1. Nucleic Acids Res 1993;21:3427-36

Rose $M$, Dütting $E$, Schröder N, Sticht $\mathrm{H}$, Brandstätter $\mathrm{JH}$, Enz R. PNUTS forms a trimeric protein complex with $\mathrm{GABA}(\mathrm{C})$ receptors and protein phosphatase 1 . Mol Cell Neurosci 2008;37:808-19

Ruma RC, Simon RA, Jeffrey RG. CAT 53: a protein phosphatase 1 nuclear targeting subunit encoded in the MHC Class I region strongly expressed in regions of the brain involved in memory, learning, and Alzheimer's disease. Brain Res Mol Brain Res 2005;138:70-83

Scaffidi P, Misteli T, Bianch ME. Release of chromatin protein HMGB1 by necrotic cells triggers inflammation. Nature 2002 418:191-5

Udho E, Tedesco VC, Zygmunt A, Krucher NA. PNUTS (phosphatase nuclear targeting subunit) inhibits retinoblastoma-directed PP1 activity. Biochem Biophys Res Commun 2002;297:463-7

Wang $\mathrm{H}$, Bloom $\mathrm{O}$, Zhang $\mathrm{M}$, Vishnubhakat JM, Ombrellino $M$, Che J, Frazier A, Yang $H$, Ivanova $S$, Borovikova $L$, Manogue KR, Faist E, Abraham E, Andersson J, Andersson U, Molina PE, Abumrad NN, Sama A, Tracey KJ. HMG-1 as a late mediator of endotoxin lethality in mice. Science 1999;285:248-51

Wilkinson B, Chen JY, Han P, Rufner KM, Goularte OD, Kaye J. TOX: an HMG box protein implicated in the regulation of thymocyte selection. Nat Immunol 2002;3:272-80 